\title{
Die vertikale Verteilung des atmosphärischen Ozons nach Ballonaufstiegen
}

\author{
Von Hans-Karl Paetzold \\ Aus dem Max-Planck-Institut für Physik der Stratosphäre, Weißenau \\ (Z. Naturforschg. 10a, 33-41 [1955]; eingegangen am 17. Dezember 1954)
}

\begin{abstract}
Durch Ballonaufstiege mit Ultraviolettspektrographen wurde in den letzten Jahren die vertikale Verteilung des atmosphärischen Ozons zu verschiedenen Jahreszeiten in Weißenau bestimmt. Die beobachteten großen Variationen müssen - vor allem unterhalb von $35-40 \mathrm{~km}$ - auf vertikale und horizontale Transportvorgänge in der Atmosphäre zurückgeführt werden. So läßt sich eine Auf- oder Abwärtsbewegung der Atmosphäre bis herauf zu $50 \mathrm{~km}$ Höhe mit einer Mindeststärke von $1-10 \mathrm{~cm} / \mathrm{sec}$ feststellen, die in $20 \mathrm{~km}$ Höhe ihr Vorzeichen umkehrt. Im Frühjahr wird durch die Zufuhr von Luft aus polaren Breiten ein sekundäres Ozonmaximum in $15 \mathrm{~km}$ Höhe bedingt. Der Jahresgang des Ozons zeigt in den verschiedenen Höhen ein unterschiedliches Verhalten, das verschiedene jahreszeitlich wechselnde Faktoren erkennen läßt, wie Photochemie des Ozons, Advektion, turbulenter Massenaustausch sowie eine Auf- und Abwärtsbewegung der Stratosphäre im Frühjahr bzw. Herbst mit einer mittleren Stärke von $0,1 \mathrm{~mm} / \mathrm{sec}$.
\end{abstract}

$\mathrm{B}_{\mathrm{i}}^{\mathrm{e}}$ ei der Erforschung des Ozonproblems wurde in den letzten Jahren die Notwendigkeit immer spürbarer, das Beobachtungsmaterial über die vertikale Ozonverteilung wesentlich zu verbreitern. Denn eine Analyse der die Ozonverteilung beeinflussenden Faktoren (photochemische Bedingungen, Lufttransport) erscheint aus den Messungen des integralen Ozonbetrages, wie sie in dem weltweiten Beobachtungsnetz mit dem DobsonSpektralphotometer erfolgen, allein kaum möglich. In dem hiesigen Institut wurden daher zur Ergänzung in den letzten Jahren drei Methoden zur Messung der vertikalen Ozonverteilung entwickelt und angewandt:

1. Ballonaufstiege mit selbstregistrierenden Ballonspektrographen,

2. Mondfinsternisse,

3. Konstruktion einer billigen und leicht zu bedienenden Radiosonde.

Während die Radiosonde noch in der Erprobung steht, konnte durch die beiden ersten Methoden schon ein breiteres Beobachtungsmaterial gewonnen werden. Die bei Mondfinsternissen gewonnenen Ergebnisse sind vom Verfasser schon früher mitgeteilt worden ${ }^{1}$. Im folgenden sollen daher die neuen Weißenauer Ballonaufstiege mit UV-Spektrographen und ihre Ergebnisse kurz dargestellt werden, wobei Einzelheiten einer ausführlicheren

${ }^{1}$ H.-K. Paetzold, Z. Naturforschg. 5a, 661 [1950]; 6 a, 639 [1951]; 7 a, 325 [1952]; J. Atm. Terr. Phys. 2, 183 [1952].
Darstellung im Rahmen der von dem hiesigen Institut herausgegebenen Veröffentlichung vorbehalten seien.

\section{Experimentelle Anordnung}

a) Aufstiegsapparatur

Es konnte an die ersten Aufstiege in Stuttgart mit UV-Spektrographen angeknüpft werden ${ }^{2}$. Da jedoch von vornherein Reihenaufstiege geplant waren, und um ein möglichst genaues Beobachtungsmaterial zu erhalten, wurde ein Aufstiegsspektrograph neu konstruiert, der die folgenden Bedingungen zu erfüllen hatte:

1. Während eines Aufstiegs waren ca. 40 Sonnenspektren zu registrieren, deren Schärfe von Aufnahme zu Aufnahme nicht merklich variieren durfte. Denn wegen der übergroßen Häufung der Frauenhoferschen Linien in dem ultravioletten Spektralbereich des Sonnenspektrums hängt die gemessene spektrale Intensitätsverteilung merklich von der Abbildungsfunktion des Spektrographen selbst ab.

2. Bei niedrigem Gewicht sollte der Spektrograph möglichst stabil sein, um eine häufige Nachjustierung oder Beschädigung bei der Landung zu vermeiden.

Um diese sich etwas widersprechenden Bedingungen zu erfüllen, wurde der Spektrograph in geschlossener Kastenbauweise konstruiert, was durch eine entsprechende Umlenkung des Strahlenganges, den Abb. 1 zeigt, erzielt wurde. Es konnte dabei auf die Kollimatorlinse verzichtet werden, da der Öffnungswinkel des das Prisma durchsetzenden Lichtbündels nur $2^{\circ}$ betrug. Die Kameralinse, eine einfache, plankonvexe Linse mit einem wirksamen Öffnungsverhältnis von
${ }^{2}$ E. u. V. H. Regener, Phys. Z. 35, 788 [1938]. 
1:15 bildete den Spalt von $3 \mathrm{~mm}$ Länge und $0,02 \mathrm{~mm}$ Breite in halber Größe ab. Vor dem Spalt befand sich ein 3-Stufen-Platinfilter zur Ableitung der Intensitätsskale der photographischen Platte. Kameralinse und das $60^{\circ}$-Prisma (20 $\mathrm{mm}$ Höhe und $30 \mathrm{~mm}$ Kantenlänge) waren aus Homosil gefertigt, das in dem benutzten Spektralbereich ( 3180 bis $2950 \AA$ ) noch keine selektive Absorption zeigte. Die lineare Dispersion betrug bei $3000 \AA 80 \AA / \mathrm{mm}$.

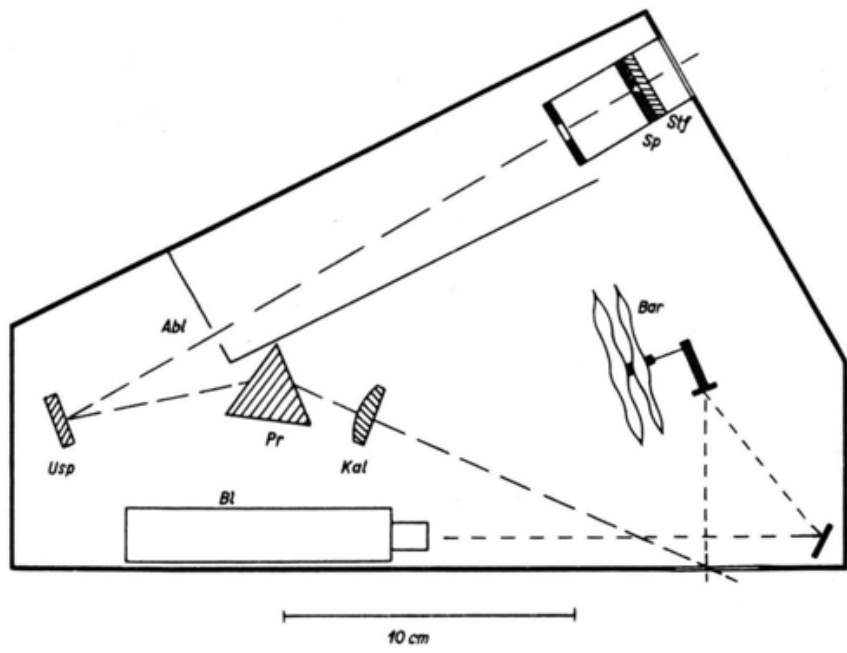

Abb. 1. Aufstiegsspektrograph. Stf: Stufenfilter, Sp: Spalt, Abl: Aperturblende, Usp: Umkehrspiegel, Pr: Prisma, Kal: Kameralinse, Bar: Barograph, Bl: Beleuchtung für den Barograph.

Als Lichtquelle diente eine von der Sonne beschienene $\mathrm{MgO}$-Scheibe, um den Spektrographen nicht auf die Sonne richten zu müssen, was für die vorliegende Zielsetzung einen zu großen experimentellen Aufwand bedeutet hätte. Bei dieser Anordnung ist aber zu beachten, daß in tieferen Luftschichten ein erheblicher Anteil der UV-Strahlung vom allgemeinen Himmelstreulicht gestellt wird, dessen spektrale Zusammensetzung nicht genau mit dem des direkten Sonnenlichtes übereinstimmt. Da das Himmelstreulicht mit zunehmender Höhe abnimmt, kann so eine Verfälschung der über dem Apparat befindlichen gemessenen Ozonschicht und damit der vertikalen Ozonverteilung bewirkt werden. Deshalb wurde durch eine Ringblende das Himmelstreulicht von der $\mathrm{MgO}$-Scheibe bis auf $40 \%$ abgeblendet. Um den restlichen Fehlereinflu $B \mathrm{zu}$ bestimmen, wurden jeweils die aus Aufnahmen mit der gewählten Anordnung und mit direktem Sonnenlicht allein folgenden Ozonwerte verglichen, wobei sich nur Unterschiede von $2-3 \%$ ergaben, die bei den Aufstiegsmessungen durch eine dem Luftdruck proportionale Korrektur berücksichtigt wurden.

Als weiteres Element befindet sich in dem Spektrographen noch die Anzeigevorrichtung für den Luftdruck sowie für die Temperatur in der Apparatur (Abb. 1). Die Meßgenauigkeit des Barographen war

${ }^{3}$ H.-K. Paetzold, Z. Angew. Phys. 6, 219 [1954]. durch die Anwendung von Federgelenken und einer optischen Registrierung so gesteigert worden, daß sie in dem gesamten zu erfassenden Druckbereich von 760 bis $3 \mathrm{~mm} \mathrm{Hg}$ ausreichte. Die Eichkurven ergaben eine reproduzierbare Genauigkeit der Druckmessung von $\pm 0,2 \mathrm{~mm} \mathrm{Hg}$, was einem Fehler in 30, 40, 45 und $50 \mathrm{~km}$ Höhe von $0,1,0,6,1,2$ und $2,5 \mathrm{~km}$ entspricht. Druck- und Temperaturmarken kommen auf der mittels Ankerhemmung, die durch eine Schaltuhr betätigt wird, alle 6,7 min bei einer Aufstiegsgeschwindigkeit von $250 \mathrm{~m} / \mathrm{min}$ schrittweise weitergedrehten Registrierplatte zwischen die UVSpektren zu liegen, so da $B$ eine sichere Zuordnung gewährleistet war. Als Aufnahmeplatten dienten Perutz-Spektralblau-Platten mit einem Auflösungsvermögen von ${ }^{3}$ ca. $100 \mathrm{~mm}^{-1}$, die 3 min in Metol-Hydrochinon-Entwickler von $17^{\circ} \mathrm{C}$ entwickelt wurden.

Zum Schutz gegen Beschädigung bei der Landung und gegen zu starke Abkühlung während des Flugs wurde der Aufstiegsspektrograph, dessen Gewicht $1 \mathrm{~kg}$ betrug, wie üblich in einer mit Kunststoffolie bespannten Gondel aufgehängt. Die Aufstiegstechnik selbst war gegenüber früher durch die neuen amerikanischen aus einem Stück geblasenen Ballone sehr vereinfacht $^{4}$. Es genügten für das Gesamtgewicht der Aufstiegsapparatur von 2,5 kg zwei Ballone mit je $2 \mathrm{~kg}$ Eigengewicht, um im Durchschnitt eine Gipfelhöhe von $31 \mathrm{~km} \mathrm{zu}$ erzielen, wobei die maximal erreichte Höhe $38 \mathrm{~km}$ betrug.

Die Höhenbestimmung erfolgte einmal barometrisch, wobei die in die barometrische Höhenformel eingehende Temperaturverteilung bis $16 \mathrm{~km}$ Höhe den Berichten des deutschen Wetterdienstes entnommen und darüber nach dem der Jahreszeit entsprechenden Mittelwert berücksichtigt wurde. Zur Kontrolle wurde bei klarer Sicht die Höhe und die horizontale Bahn des Ballongespannes durch Messung von Azimut und Höhenwinkel (Theodolith) sowie des Sehwinkels, unter dem die Basis Ballon - Gondel $(50 \mathrm{~m})$ erschien, (Spiegelteleskop, Okularmikrometer) bestimmt. Die Differenz in der Höhenmessung zwischen beiden Verfahren betrug bis $30 \mathrm{~km}$ Höhe und darüber nie mehr als 0,5 bis $1 \mathrm{~km}$, so daß der Höhenfehler nicht größer als $\pm 0,5 \mathrm{~km}$ sein wird. Die sich daneben aus der horizontalen Flugbahn ergebenden Windgeschwindigkeiten und Windrichtungen in den verschiedenen Höhen waren für die Diskussion einzelner gemessener Ozonverteilungen von Bedeutung.

Wegen der extremen Lage Weißenaus im Süden der Bundesrepublik wurden bei ungünstiger Windrichtung, bei der ein Abtreiben des Ballongespannes in die Alpen zu befürchten war, die Aufstiege ca. $60 \mathrm{~km}$ nördlich von Weißenau durchgeführt. Dies war notwendig, um eine einseitige Auswahl in bezug auf die advektiven Verhältnisse in der Atmosphäre zu vermeiden. Die Verlustquote konnte auf diese Weise sehr klein gehalten werden, so da $\beta$ bei insgesamt 18 Aufstiegen nur eine Aufstiegsapparatur verlorenging.

${ }^{4}$ Lieferfirma: Dewalco, Cambridge, USA. 


\section{b) Auswertung}

Aus den in verschiedenen Höhen $h$ aufgenommenen Spektren ist der Intensitätsabfall an ihrem ultravioletten Ende, unterhalb von $3200 \AA$, der wesentlich durch die spektrale Ozonabsorption im Bereich der sog. Hartley-Bande bedingt wird, zu bestimmen und daraus die Dicke $x(h)$ der in der Höhe $h$ jeweils über der Apparatur liegenden Ozonschicht abzuleiten. Da die vertikale Ozonverteilung $\varepsilon(h)\left[\mathrm{cm} \mathrm{O}_{3} / \mathrm{km}\right]$ durch Differentiation aus der integralen Kurve $x(h)$ folgt, mußte letztere möglichst genau gemessen werden, damit auch für geringere Höhen unterhalb von $10-15 \mathrm{~km}$, wo $x(h)$ nur langsam abnimmt, die Fehler in $\varepsilon(h)$ nicht zu groß werden. Es seien $I(\lambda, h), I_{0}(\lambda)$ die spektralen Intensitäten des Sonnenlichtes in der Höhe $h$ bzw. extraterrestrisch, $k_{\mathrm{R}}(\lambda)$ die dekadische RayleighExtinktion für die Luftmasse 1, $M(z, h)$ die durchstrahlte Luftmasse für die Zenitdistanz $z$ und $\alpha(\lambda)$ der dekadische Absorptionskoeffizient des Ozons. Dann gilt die hier vereinfachte Grundgleichung:

$$
I(\lambda, h)=I_{0}(\hat{\lambda}) \cdot 10^{-\sec z \cdot \alpha(\lambda)} \cdot 10^{-M(z) k_{\mathrm{R}}(\lambda)} .
$$

In ihr ist die Dunstextinktion vernachlässigt worden, da sie sich nur in Höhen unterhalb von $3 \mathrm{~km}$ merklich auswirken wird. Ferner ist in dem ersten Term der rechten Seiten der Einfluß der atmosphärischen Refraktion und der Krümmung der atmosphärischen Schichten nicht berücksichtigt. Er wird erst für eine Zenitdistanz $z$ oberhalb von $70^{\circ}$, wo er $2 \%$ beträgt, merklich und brauchte daher nur für einige Winteraufstiege berücksichtigt zu werden.

Für die Auswertung geht nur der relative spektrale Intensitätsverlauf ein. Der feste Bezugspunkt $\lambda_{0}$ wurde bei der Frauenhofer-Linie $R$ $(3179 \AA)$ gewählt, d. h. dicht bei dem von der Ozonabsorption stark beeinflußten Spektralbereich, um sekundäre Einflüsse wie Rayleigh-Extinktion und die Selektivität der Aufnahmeapparatur möglichst klein zu halten. Führt man statt der Wellenlänge $\lambda$ den Absorptionskoeffizienten des Ozons $\alpha$ ein und seien $I^{\prime}(\alpha, h)$ die auf der Platte gemessenen Intensitäten, $R\left(\alpha, \alpha_{0}, h\right)$ und $S\left(\alpha, \alpha_{0}\right)$ die Korrekturglieder für die Rayleigh-Extinktion und die Selektivität des Spektrographen und der

${ }^{5}$ H.-K. Paetzold, Z. Naturforschg. 2a, 219 [1947]; Optik 6, 327 [1950].
Photoplatte, dann folgt aus Gl. (1) für die Auswertung:

$$
\begin{array}{r}
\log \frac{I^{\prime}(\alpha, h)}{I^{\prime}\left(\alpha_{0}, h\right)}-\log \frac{I_{0}(\alpha)}{I_{0}\left(\alpha_{0}\right)}+R\left(\alpha, \alpha_{0}, h\right)+S\left(\alpha, \alpha_{0}\right) \\
=F(\alpha, h), \\
\quad(2 \mathrm{a}) \\
F(\alpha, h)=-\sec z \cdot x(h) \cdot\left(\alpha-\alpha_{0}\right) .
\end{array}
$$

Gl. (2 b) stellt eine graphisch einfach zu konstruierende Gerade dar, aus deren Neigung das gesuchte $x(h)$ folgt. Die Rayleigh-Korrektur ist nur unterhalb von $10 \mathrm{~km}$ merklich. Das selektive Glied $S\left(\alpha, \alpha_{0}\right)$ ist ebenfalls nicht sehr groß, wie entsprechende Vergleichsmessungen mit einem $\mathrm{H}$ Kontinuum ergaben. Denn die Empfindlichkeit der photographischen Emulsion sowie die Reflexionsfähigkeit des Aluminiumspiegels und der $\mathrm{MgO}$-Scheibe sinken in dem schmalen benutzten Spektralbereich von höchstens $200 \AA$ Breite nur jeweils um 1-2\% ab. Etwas stärker ist die spektrale Abhängigkeit der Platin-Stufenfilter. Zur Eichung der drei Durchlässigkeitsstufen von 100, 40 und $14 \%$, die zur Herstellung der Intensitätsskala für die photographische Platte dienten, wurde ein vom Verfasser früher entwickeltes photometrisches Verfahren benutzt, das auf der Beugung am Spalt beruht und besonders im ultravioletten Spektralbereich von Vorteil ist ${ }^{5}$. Bei einem der verwendeten Filter ergab sich bei der geringsten (III.) Durchlässigkeitsstufe von 3180 bis $2950 \AA$ ein Absinken der Transparanz von 11,0\% auf 9,5\% und ein Anstieg des Transparanzverhältnisses zwischen der II. und III. Stufe von 4,85 auf 5,53.

Am unsichersten und problematischsten ist in Gl. (2) das Glied $\log \left\{I_{0}(\alpha) / I_{0}\left(\alpha_{0}\right)\right\}$, das die extraterrestrische spektrale Intensitätsverteilung des Sonnenlichtes berücksichtigt. Von der letzteren liegen zwar jetzt Messungen durch die amerikanischen Raketenaufstiege ${ }^{6}$ vor, doch ist eine Utbertragung derselben wegen der starken Häufung der Frauenhofer-Linien und der unterschiedlichen Apparatefunktionen nicht ohne weiteres möglich. Es zeigte sich, daß mit der Intensitätsverteilung nach den Raketenaufstiegen im Bereich zwischen 3180 und $3060 \AA$, d. h. von $\alpha=0,5 \mathrm{~cm}^{-1}$ bis $\alpha=2,5 \mathrm{~cm}^{-1}$ sich die einzelnen Meßpunkte gut auf der Geraden $F(\alpha)$ anordneten und die daraus folgenden Ozonwerte $x(h)$ am Erdboden befriedigend mit den in dem $100 \mathrm{~km}$ von Weißenau entfernten Arosa mit

\footnotetext{
${ }^{6}$ The Atmospheres of the Earth a. Planets, ed. by G. I. Kuiper, p. 125, Chicago Press 1952.
} 
dem Dobson-Spektrographen gemessenen Werten übereinstimmten. Unterhalb von $3060 \AA$ ergaben sich aber im Vergleich zu der amerikanischen Intensitätsverteilung systematische Abweichungen. Daher wurde für unsere Aufstiegsapparatur für diesen Spektralbereich eine individuelle Intensitätsverteilung abgeleitet, indem dieselbe so variiert wurde, daß für $\lambda<3060 \AA$ die Meßpunkte auf der Geraden $F(\alpha)$ lagen. So konnten die Aufnahmen für größere Höhe und damit größere Werte von $\alpha$ sukzessive aneinander angeschlossen werden. Die Resultate von mehreren Aufstiegen wurden zu einer ,Standard“-Intensitätsverteilung gemittelt, die dann für alle Aufstiege zugrunde gelegt wurde.
$0,3 \cdot 10^{-3}\left[\mathrm{~cm} \mathrm{O}_{3}\right] / \mathrm{km}$. Da bei den bis jetzt vorliegenden Aufstiegen ca. 600 Spektren mit je drei Intensitätsstufen auszumessen waren, war das ganze Auswerteverfahren weitgehend rationalisiert worden.

\section{Ergebnisse und Diskussion}

In Abb. 2 sind die bei den bisherigen Aufstiegen gemessenen integralen Kurven $x(h)$ dargestellt, die über alle Jahreszeiten verteilt sind. Wegen der jahreszeitlichen Abhängigkeit des Ozons, dessen totaler Betrag bekanntlich in mittleren Breiten im Frühjahr ein Maximum und im Herbst ein Minimum aufweist ${ }^{7}$, sind die Beobachtungsergebnisse
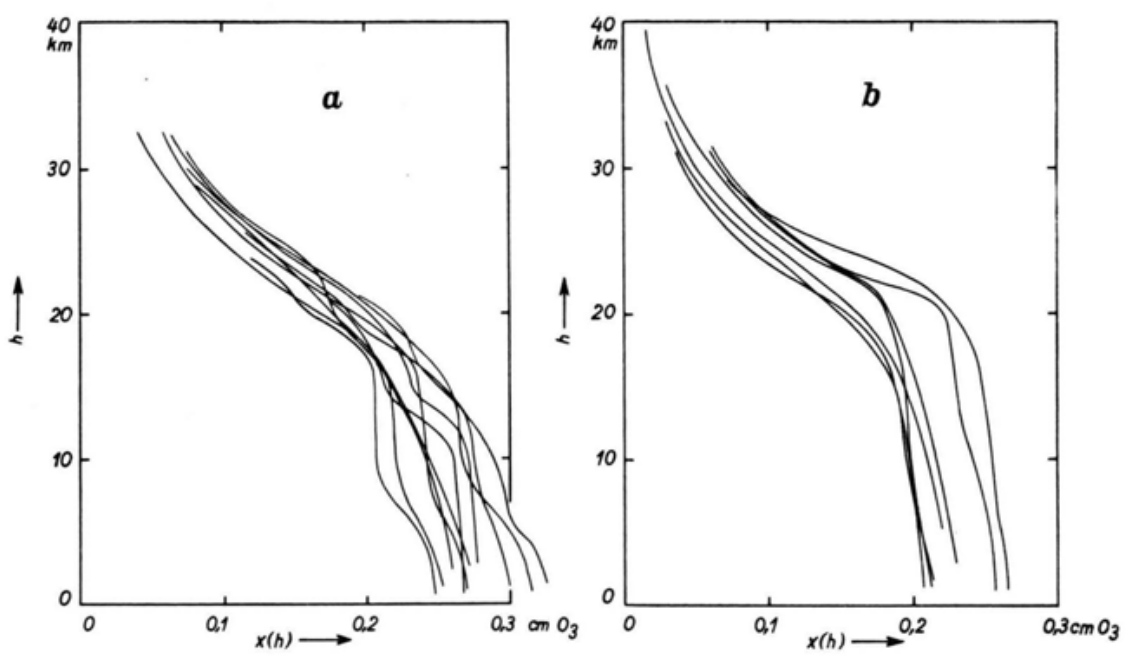

Abb. 2. Die gemessenen integralen Kurven $x(h)$. a) Frühjahr. Sommer; b) Herbst, Winter.

Die Spektren wurden mit einem Zeiss'schen Registrierphotometer photometriert. Um eine hohe innere Genauigkeit zu erzielen, mußten die Enveloppen streng einheitlich an die Photometerkurven gelegt werden. Zur Prüfung diente ein schon früher angegebenes Verfahren ${ }^{5}$, bei dem sich für die drei Intensitätsstufen drei ,,Schwärzungskurven" ergeben, die einander streng parallel in Abständen der Logarithmen der Durchlässigkeiten der Filterstufen verlaufen müssen. Dadurch hätte insbesondere ein evtl. langwelligerer Streulichtanteil erfaßt werden können, der aber immer unter einer nachweisbaren Grenze blieb. Der gesamte Fehler in den Ozonwerten $x(h)$ betrug bis zu den größten erreichten Höhen, wie eine Reihe von Vergleichen zeigte, nur $1-2 \%$. Für die differenzierten Kurven $\varepsilon(h)$ ergab sich in 5,25 und $30 \mathrm{~km}$ Höhe eine Unsicherheit von $\pm 1 \cdot 10^{-3}, 0,5 \cdot 10^{-3} \mathrm{bzw}$. für Frühling, Sommer und Herbst, Winter getrennt gegeben. Es fällt sofort auf, daß die Kurven im Frühjahr zwischen 0 und $20 \mathrm{~km}$ Höhe wesentlich stärker variieren als im Herbst. Hier deutet sich für die differenzierte Kurve $\varepsilon(h)$, d. h. die Ozonverteilung, ein zweites Maximum in ca. $15 \mathrm{~km}$ Höhe neben dem ersten bekannten photochemisch bedingten in $23 \mathrm{~km}$ Höhe an. Dabei ist im Mittel der totale Ozonbetrag im Frühjahr deutlich größer als im Herbst, wie es dem obigen, mit dem DobsonSpektralphotometer festgestellten Jahresgang entspricht. Ferner weist aber auch der über $30 \mathrm{~km}$ Höhe befindliche Ozonbetrag erhebliche Schwankungen auf, die hier prozentual mit am stärksten sind und die weit außerhalb der Meßgenauigkeiten liegen.

${ }^{7}$ F. W. P. Götz, Compendium of Meteorology, p. 275,1950 . 
Diese starken Schwankungen der integralen Kurven und damit auch der vertikalen Ozonverteilung entsprechen ganz den Ergebnissen früherer Ballonaufstiege und der Methode der Mondfinsternisse ${ }^{8,9}$. Bei der Klassifikation der beobachteten Ozonverteilungen können mehrere in Abb. 3 dargestellte Haupttypen unterschieden werden, zwischen denen natürlich Übergangsformen bestehen:

Type I: 1 Maximum in ca. $23 \mathrm{~km}$ Höhe

(Kurve 1 u. 2),

Type IIa: 2 Maxima in ca. 23 und $15 \mathrm{~km}$ Höhe

(Kurve 4),

Type IIb: 2 Maxima in ca. 23 und $6 \mathrm{~km}$ Höhe (Kurve 3),

Type III: 3 Maxima in ca. 23, 15 und $6 \mathrm{~km}$ Höhe (Kurve 5).

In bezug auf das erste Maximum in $23 \mathrm{~km}$ Höhe ist weiterhin die Unterscheidung in spitze oder flache Formen von Bedeutung (Kurve 1 u. 2). Ferner sind die beiden Maxima in 23 und $15 \mathrm{~km}$ Höhe nicht immer so diskret abgesetzt wie in Kurve 4, sondern verlaufen oft miteinander zu einem breiten Buckel.

In Anbetracht der sehr großen Vielfalt der auftretenden Ozonverteilungen ist es nicht überraschend, daß die vorliegenden zahlreichen Messungen des totalen Ozonbetrages vom Boden aus mit dem Dobson-Spektralphotometer bislang keine befriedigende Erfassung der einzelnen wirksamen Mechanismen zugelassen haben. Denn der totale Ozonbetrag wird sich nach Abb. 3 in komplizierterer Weise aus den Teilbeträgen in den verschiedenen Höhenbereichen zusammensetzen, die ihrerseits von den einzelnen Faktoren unterschiedlich bedingt sein werden. Als diese sind zu nennen:

1. die photochemische Bildung und Zersetzung des Ozons,

2. vertikale und horizontale Transportvorgänge in der Atmosphäre,

3. Oxydationsprozesse, die aber in dem hier betrachteten Höhenbereich oberhalb von $3 \mathrm{~km}$ im Durchschnitt nur von untergeordneter Bedeutung sein werden.

Nach der photochemischen Theorie ist nun die Zeit, in der sich bei Änderungen der photochemischen Faktoren der neue photochemische Gleichgewichtszustand wiedereinstellt in 40,30 und

${ }^{8}$ H.-K.Paetzold, Ber. Deutsch. Wetterd. US-Zone, Nr. 38, 292 [1952].

9 E. Regener, J. Atm. Terr. Phys. 2, 173 [1952].
$20 \mathrm{~km}$ Höhe von der Größenordnung $10 \mathrm{Stdn}$., 10 Tage bzw. 3 Jahre ${ }^{10,11}$. Danach können die interdiurnen Ozonschwankungen unterhalb von etwa $40 \mathrm{~km}$ Höhe keinesfalls durch Änderungen der photochemischen Bedingungen (Temperatur oder UV-Strahlung) bewirkt werden. Unterhalb dieser Höhe ist vielmehr das Ozon ein weitgehend konservatives Element eines Luftkörpers, worauf seine Rolle als Indikator für horizontale und vertikale Luftbewegungen beruht. So müssen z. B. die in Abb. 2 sichtbaren großen einzelnen Schwankungen des Ozonbetrages oberhalb von $30 \mathrm{~km}$ im wesentlichen durch Transportvorgänge in der Atmosphäre erzeugt werden. Ferner kann unterhalb von ca. $28 \mathrm{~km}$ Höhe das Ozon photochemisch nicht mehr den durch den jährlichen Gang der Sonnenhöhe veränderten Einstrahlungsbedingungen des ultravioletten Sonnenlichtes folgen, so da $\beta$ die markanten Unterschiede der Ozonverteilung zwischen Herbst und Frühling unterhalb von $20 \mathrm{~km}$ Höhe ebenfalls auf Luftbewegungen zurückzuführen sind.

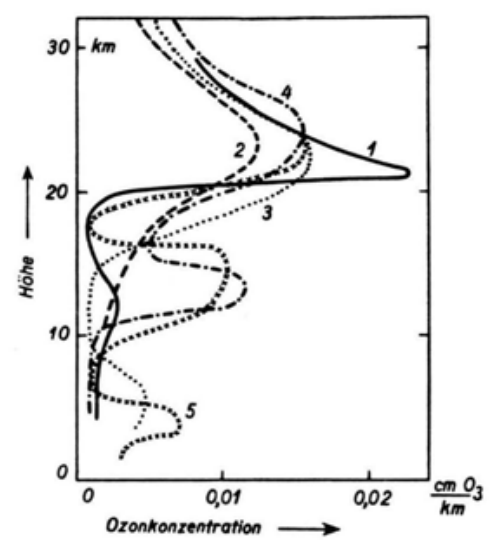

Abb. 3. Verschiedene Typen der vertikalen Ozonverteilung in Weißenau $\left(48^{\circ} \mathrm{N}\right)$. 1 : 8.9.1953; 2: 23.10. 1953 ; 3: 18.3.1953; 4 : 20.4.1953; 5: 18.4. 1952 .

Bei dem vertikalen Lufttransport sind zwei Arten zu unterscheiden: einmal auf- und absteigende Luftströmungen, zum andern der turbulente Massenaustausch. Der letztere versucht überall das gleiche Partialdruckverhältnis Ozon/Luft herzustellen, während bei den ersteren dieses erhalten bleibt. Der letztere Effekt ist bei den beiden bis zu $38 \mathrm{~km}$ Höhe reichenden Herbstaufstiegen vom

${ }^{10}$ H.-K. Paetzold, Geophys. pure et appliqué $\mathbf{2 4}$, 1 [1953].

${ }_{11}$ H. U. Dütsch, Diss. Zürich 1946. 
28.9. und 23.10.53 (Abb. 4) festzustellen, bei denen oberhalb von $20 \mathrm{~km}$ sich zwei Maxima der Kurven Ozon/Luft andeuten, die aber in verschiedenen Höhen liegen ${ }^{12}$. Ein gleiches Verhalten zeigen auch unsere Ergebnisse der Mondfinsternisse nach Abb. 5, die für äquatoriale Gegenden gelten, und die bei amerikanischen Raketenaufstiegen gewonnenen Ergebnisse ${ }^{13}$. Diese Beobachtungen lassen mit großer Wahrscheinlichkeit auf den Einfluß vertikaler Luftströmungen schließen. Für ihre Geschwindigkeiten ergibt sich aus der Einstellzeit des
Vertikalbewegungen der Atmosphäre erklären, wie Abb. 5 demonstriert, den oben erwähnten auffälligen Wechsel zwischen spitzen und flachen Formen des primären Ozonmaximums zwanglos, was durch den Massenaustausch kaum möglich erscheint. Falls letzterer die vertikalen Ozonverteilungen maßgeblich variieren soll, müßte der sog. Austauschkoeffizient in $30 \mathrm{~km}$ von der Größenordnung $1 \mathrm{~g} / \mathrm{cm} \cdot \mathrm{sec}$ sein, während er im Durchschnitt zu $1 \cdot 10^{-2} \mathrm{~g} / \mathrm{cm} \cdot \mathrm{sec}$ anzunehmen ist $^{14}$, wenn auch temporär größere Werte vorkommen mögen ${ }^{8}$. Eine
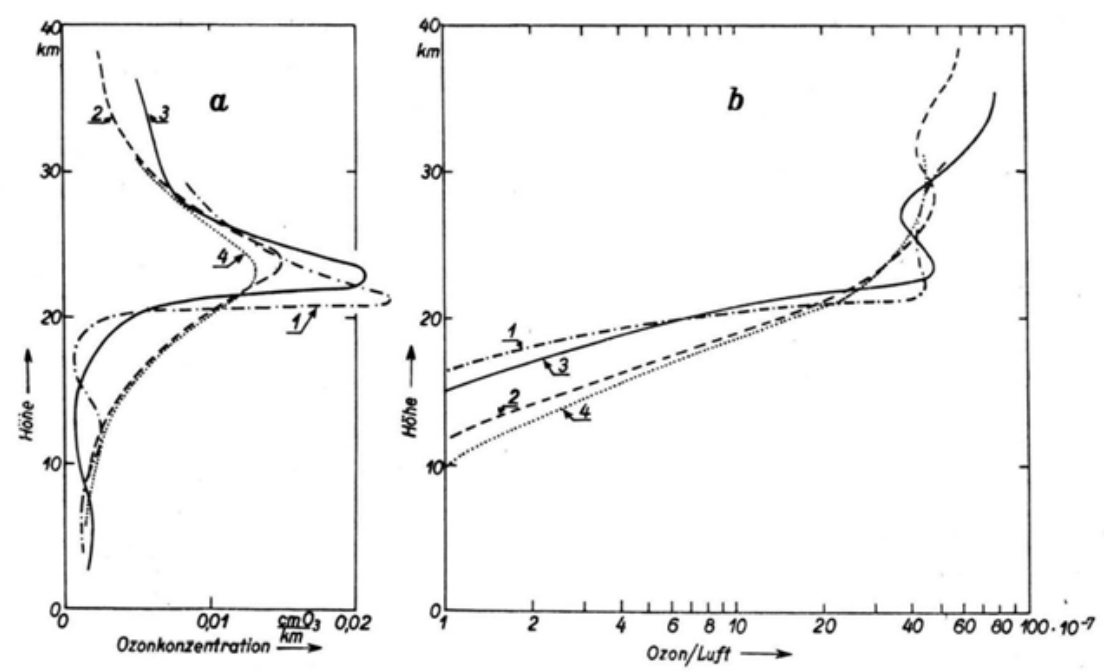

Abb. 4. Ozonverteilungen im Herbst 1953 in Weißenau. 1: 8.9.1953; 2: 28.9.1953; 3: 23.10.1953; 4 : 28.10 .1953$.

photochemischen Gleichgewichtes und aus dem zeitlichen Abstand zweier Aufstiege am 2. und 8. 9. 53, die dieselbe Erscheinung zeigen, für $30 \mathrm{~km}$ Höhe eine untere Grenze von $1 \mathrm{~cm} / \mathrm{sec}$. Aus den bei den Raketenaufstiegen bis ca. $50 \mathrm{~km}$ Höhe festgestellten Variationen würde, falls diese sich bestätigen sollten, eine vertikale Minimalgeschwindigkeit von $10 \mathrm{~cm} / \mathrm{sec}$ folgen.

Auffällig ist nun in Abb. 4 u. 5, daß sich die Kurven Ozon/Luft in ea. $20 \mathrm{~km}$ schneiden, d. h. unterhalb dieser Höhe verlaufen diese Vertikalbewegungen entgegengesetzt, so daß es sich um eine Art Schwingungsvorgang handeln würde, wie er ähnlich schon früher bei den sogenannten Föhnwogen gefunden worden ist. Diese gegenläufigen

${ }^{12}$ H.-K. Paetzold, Naturwiss. 41, 318 [1954].

${ }^{13}$ F. S. Johnsen, J. D. Purcell, R. Tousey u. K. Watanabe, J. Geophys. Res. 57, 157 [1952].

${ }^{14}$ H.-K. Paetzold, Geofis. Pura Appl., erscheint demnächst.
Wirkung des turbulenten Massenaustausches erscheint jetzt gesichert, der Transport des in der Troposphäre und am Erdboden festgestellten Ozons aus Höhen um $20 \mathrm{~km}$, wo es photochemisch nachgebildet wird ${ }^{15,16}$. Für diesen Vorgang lassen die neuen und die früheren Messungen der Ozonverteilung in Verbindung mit den jetzt sichereren Grundlagen der photochemischen Theorie auch eine quantitativ befriedigende Darstellung zu, wonach sich ein dauernder von oben nach unten gerichteter Ozonstrom mit einer durchschnittlichen Stärke von $1 \cdot 10^{11}\left[\mathrm{O}_{3}\right.$-Molek./ $\left.\mathrm{cm}^{2} \mathrm{sec}\right]$ ergibt ${ }^{14},{ }^{17}$.

Für den horizontalen Lufttransport ergab sich mit dem Auftreten des sekundären Maximums in ca. $15 \mathrm{~km}$ Höhe in Abb. 3 nach unseren Aufstiegen

15 E. Regener, Meteor. Z. 60, 253 [1943].

${ }^{16}$ H.-K. Paetzold, Geofis. Pura Appl. 24, 15 [1953].

${ }^{17}$ H.-K. Paetzold, J. Atm. Terr. Phys., erscheint demnächst. 
jetzt eine sichere Korrelation. Denn nach der Konstruktion der Luftbahnen in 11 und $16 \mathrm{~km}$ Höhe nach den Höhenwetterkarten, wie sie für alle Aufstiege durchgeführt wurden, ergab sich ausnahms. los, da $\beta$ immer, wenn die Luft aus polaren Breiten stammt, dieses Maximum im Frühjahr auftritt. Dagegen ist der Ozonbetrag zwischen 10 und $20 \mathrm{~km}$ Höhe gering, wenn die Luft in dieser Höhe aus subtropischen Breiten stammt (Kurve 3 in Abb.3).

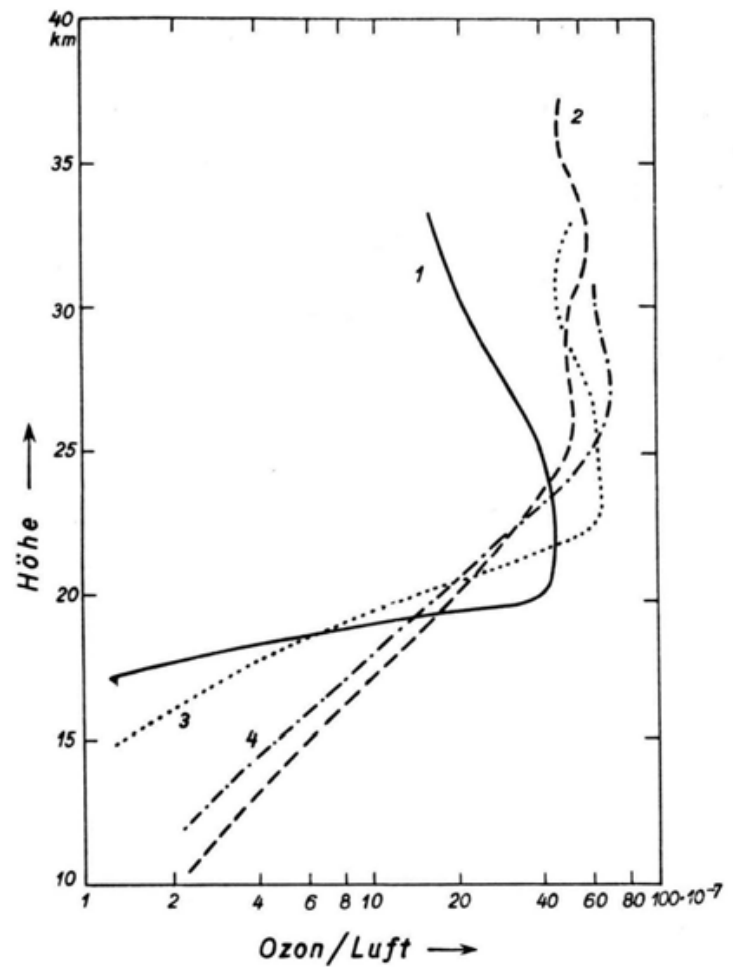

Abb. 5. Äquatoriale Ozonverteilungen nach Mondfinsternissen $1: 2.3 .1942 ; 2$ : 29.9. 1941; 3: 14.9.1932; 4: 15.8 .1943 .

Die Deutung des sekundären Ozonmaximums durch Advektion wird durch die Beobachtung erhärtet, daß sein Auftreten verknüpft ist mit einem Umspringen des Windes in der betreffenden Höhe, wie es Abb. 6 an einem Beispiel demonstriert.

Dieses sekundäre Maximum tritt im Laufe des Jahres immer schwächer auf, wie die Abb. 7 anschaulich zeigt, bei der für alle vier beobachteten Ozonverteilungen die Luft in $15 \mathrm{~km}$ Höhe aus Grönland stammte. Dieses Verhalten stimmt gut mit den jahreszeitlichen Variationen der vertikalen Ozonverteilungen in polaren Breiten überein, wie sie indirekt aus Messungen des sog. Umkehreffektes mittels des Dobson-Spektralphotometers er- schlossen wurden ${ }^{18}$. Danach wird im Frühjahr bis herab zu $10 \mathrm{~km}$ Höhe eine hohe Ozonkonzentration von $1,2 \cdot 10^{-2}\left[\mathrm{~cm} \mathrm{O}_{3} / \mathrm{km}\right]$ beobachtet, während diese im Herbst unterhalb von $20 \mathrm{~km}$ Höhe stark
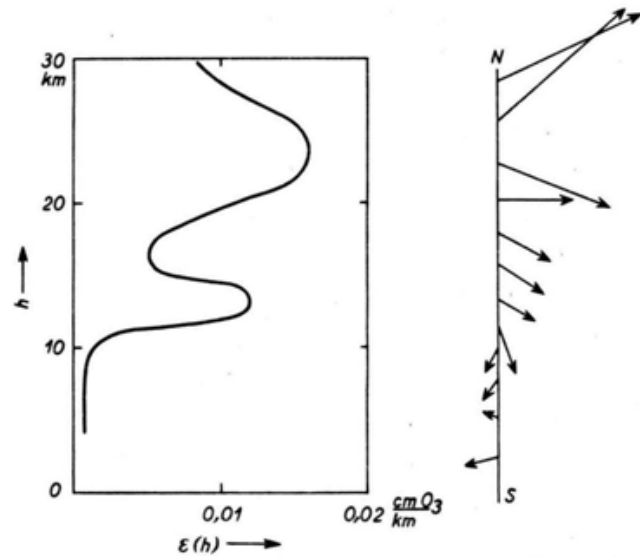

Abb. 6. Sekundäres Ozonmaximum und vertikale Windverteilung (20.4. 1953). Die Länge der Pfeile gibt die Windgeschwindigkeit $0,3 \mathrm{~mm}=5 \mathrm{~km} / \mathrm{sec}$.
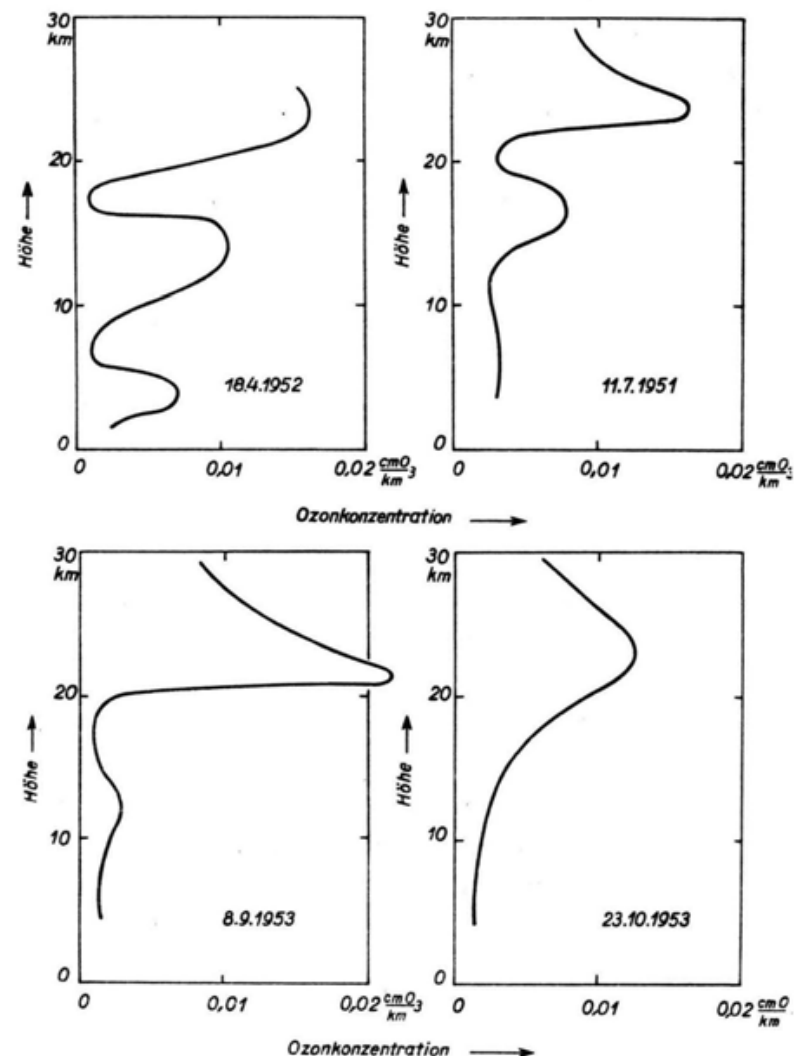

Abb. 7. Die jahreszeitliche Abhängigkeit des advektiven sekundären Ozonmaximums in $15 \mathrm{~km}$ Höhe. Die Luft in dieser Höhe stammt einheitlich aus Grönland.

18 E. Tönsherg u. K. L. Ohlsen, Geophys. Pol. 13, Nr. 12 [1944]. 
abfällt, wie es bei den mittleren und äquatorialen Breiten der Fall ist. Dieses tiefere polare Ozon muß aus größeren Höhen oberhalb von $30 \mathrm{~km}$ in geringere herabtransportiert werden, wo es sich photochemisch geschützt ansammeln kann, was sich am einfachsten durch photochemische Nachbildung des Ozons oberhalb von $30 \mathrm{~km}$ deuten läßt. Doch besteht auch die Möglichkeit, daß es aus niederen Breiten durch eine Großraumzirkulation herbeigeführt wird ${ }^{19}$. Zwischen beiden Annahmen kann heute noch nicht sicher entschieden werden, vielleicht sind beide Effekte wirksam. Auf jeden Fall muß für den vertikalen Ozontransport der Austauschkoeffizient in Höhen um $30 \mathrm{~km}$ temporär stark erhöht sein bis auf den Wert von $5 \mathrm{~g} / \mathrm{cm} \cdot \mathrm{sec}$. Dies läßt sich plausibel durch den großen horizontalen Temperaturgradienten deuten, wie er an der Grenze der Polarnacht in der ,warmen “ Schicht in 45 bis $50 \mathrm{~km}$ Höhe herrschen muß, die durch ultraviolette Ozonabsorption aufgeheizt wird ${ }^{20}$. Im Laufe des Jahres wird dann dieses Reservoir des tieferen polaren Ozons allmählich erschöpft, so daß das sekundäre Ozonmaximum im Herbst in mittleren Breiten nicht mehr beobachtet wird.

Die beobachtete jahreszeitliche Variation des sekundären Ozonmaximums leitet über zu dem Jahresgang des totalen Ozonbetrages. Nach dem Vorangegangenen ist anzunehmen, daß sich dieser ebenfalls in komplizierterer Weise zusammensetzen wird. Deshalb wurde aus unseren Messungen und denen anderer Autoren bei Ballon- und Raketenaufstiegen ${ }^{13,21,22,23}$ für mittlere Breiten die gemittelte vertikale Ozonverteilung abgeleitet, wie sie in Abb. 8 wiedergegeben ist. Aus ihr sowie aus der Zusammenstellung der einzelnen Messungen der in verschiedenen Höhen befindlichen Teilozonbeträge $^{12,24}$ ergibt sich, daß die letzteren jahreszeitlich verschiedene Variationen aufweisen: Zwischen 10 und $20 \mathrm{~km}$ Höhe wird ein Maximum im Frühjahr beobachtet, das vornehmlich von dem advektiven, sekundären Maximum bedingt wird. Sein mittlerer Betrag ergibt sich zu $0,04 \mathrm{~cm} \mathrm{O}_{3}$. Oberhalb von $30 \mathrm{~km}$ Höhe zeigt sich ein Maximum im Sommer an mit einer Amplitude von $0,02 \mathrm{~cm} \mathrm{O}_{3}$,

${ }^{19}$ H. U. Dütsch, UGGI-Tagung, Rom 1954.

${ }_{20}$ H. Moser, Ber. Deutsch. Wetterd. US-Zone, Nr. 11, 28 [1949].

21 V. H. Regener, Z. Phys. 109, 642 [1938]; Nature, Lond. 167, 276 [1951]. das sehr wahrscheinlich durch den hohen Sonnenstand erzeugt wird, da in diesen Höhen das Ozon dem jährlichen Gang desselben photochemisch folgen kann. Zwischen 20 und $25 \mathrm{~km}$ deutet sich im Sommer ein Minimum des dort befindlichen Ozonbetrages an. Die plausibelste Ursache ist die im Sommer höher reichende Konvektion, durch die aus diesen Schichten mehr Ozon nach unten geschafft wird. Es genügt die plausible Annahme, da $\beta$ im Sommer in $15 \mathrm{~km}$ Höhe der Austauschkoeffizient doppelt so groß ist als der Jahresdurchschnitt. Zwischen 25 und $30 \mathrm{~km}$ Höhe zeigt der Ozonbetrag keine ins Auge fallenden jahreszeitlichen Variationen. Offenbar reichen in diesem Höhenbereich die photochemischen bzw. konvektiven Einflüsse nicht mehr in genügender Stärke hinab bzw. hinauf oder gleichen sich weitgehend aus. Dieser Bereich ist noch weiter dadurch ausgezeichnet, daß hier die Streuung der einzelnen gemessenen Ozonbeträge am geringsten ist.

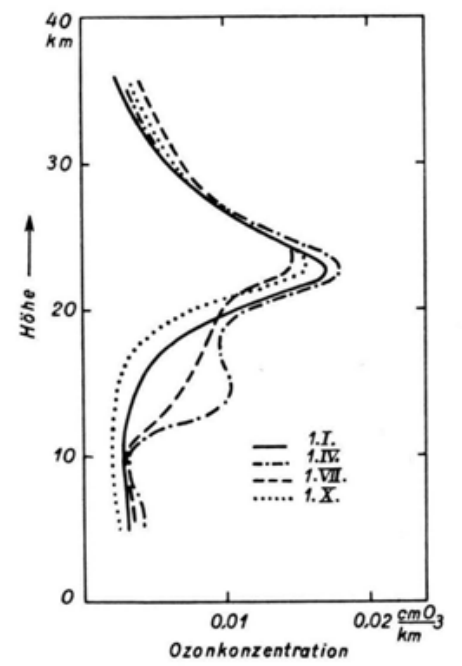

Abb. 8. Die jahreszeitliche Variation der vertikalen Ozonverteilung in mittleren Breiten.

Durch diese drei Faktoren, Photochemie, Advektion und turbulenter Massenaustausch, ist aber der in mittleren Breiten beobachtete Ozonjahresgang allein nicht zu erklären, da einmal mit ihnen allein der schon Anfang November wiedereinset-

${ }^{22}$ W. W. Coblentz u. R. Stair, J. Res. Nation Bur. Stand. 22, 573 [1939].

${ }^{23}$ J. A. van Allen u. J. D. Hopfield, Mém. Soc. roy. soc. Liége 4 . Ser. XII, 179 [1952].

${ }_{24}$ H.-K. Paetzold, Mitt. Deutsch. Wetterd., im Erscheinen. 
zende Anstieg des totalen Ozonbetrages sich nicht plausibel deuten läßt. Zum andern ergibt sich auch eine zu geringe Amplitude des Ozonjahresganges. Es muß vielmehr noch ein vierter Faktor angenommen werden, der sich aus dem jahreszeitlichen Verlauf des Partialdruckverhältnisses Ozon/Luft mit der Höhe ergibt ${ }^{17}$. Danach verläuft die Kurve Ozon/Luft für den 1. Januar zwischen 10 und $25 \mathrm{~km}$ Höhe parallel zu der am 1. Oktober, nur um $1 \mathrm{~km}$ tiefer versetzt. Dies läßt darauf schließen, daß in diesem Zeitraum die Stratosphäre im Mittel um diesen Betrag abgesunken ist und durch eine entsprechende Kompression des Ozons der Herbstanstieg desselben verursacht wird. Im Frühjahr ist eine entgegengesetzte Luftströmung anzunehmen. Die mittlere Stärke dieser ab- bzw. aufsteigenden Strömung, die - wie hier nicht näher ausgeführt sei - als Wirkung der im Herbst verstärkten Tiefdrucktätigkeit aufzufassen ist, ergibt sich $\mathrm{zu}$ $0,1 \mathrm{~mm} / \mathrm{sec}$. Sie muß in diesem Höhenbereich verbunden sein mit einem horizontalen Lufttransport von der Sommer- zur Winterhalbkugel der Erde in der Größenordnung $1 \mathrm{~m} / \mathrm{sec}$.

Im Gegensatz zum Jahresgang des Ozons ist für eine detailliertere Erfassung der interdiurnen Variationen der vertikalen Ozonverteilung, die für die Wetterprognose von Bedeutung sein kann, das vorliegende Beobachtungsmaterial noch zu klein. Ein markantes Beispiel für die Zusammenhänge zwischen Ozonverteilung und Luftbahnen hat sich oben bei dem wechselnden Auftreten des sekundären Ozonmaximums im Frühjahr ergeben. Allgemein kann abgeschätzt werden, daß im Frühjahr die interdiurnen Ozonvariationen etwa je zur Hälfte durch Advektion und vertikale Luftbewegungen verursacht werden. Im Herbst dagegen werden die letzteren ausschlaggebend sein. Diese Annahme wird auch durch eine neue Analyse der Beobachtungen des totalen Ozonbetrages mit dem Dobson-Spektralphotometer gestützt ${ }^{25}$.

Die vorliegenden neuen Messungen der vertikalen Ozonverteilung ermöglichen bereits eine etwas eingehendere Analyse der einzelnen wirksamen Faktoren. Dabei ist besonders auf den obigen direkten Nachweis von vertikalen Luftströmungen sehr geringer Stärke hinzuweisen, wie er anders wohl nur indirekt statistisch möglich wäre. Für die hier angeschnittenen Probleme der Großraumzirkulation ist in Zukunft die direkte Messung der vertikalen Ozonverteilung in verschiedenen geographischen Breiten notwendig. Hier sind die polaren Breiten sowie die Zonen der jet-streams von besonderem Interesse. Für derartige Messungen sowie für die nähere Erfassung der interdiurnen Variationen der vertikalen Ozonverteilung ist der Einsatz einer Ozonradiosonde erforderlich, deren Ergebnisse an die Messungen mit UV-Spektrographen anzuschließen sind, wie es von uns geplant ist. Es ist zu erwarten, daß derartige Messungen auf breiterer Basis in Verbindung mit den laufenden Messungen des totalen Ozonbetrages mit dem Dobson-Spektrograph bald zu einer befriedigenden Erfassung des Ozonproblems führen werden.

Ich danke Herrn Prof. E. Regener für die stete Förderung der vorliegenden Untersuchungen. Der Deutschen Forschungsgemeinschaft sei auch an dieser Stelle für Bereitstellung von apparativen Mitteln gedankt.

${ }^{25}$ Ch. Normand, Quart. J. R. Meteor. Soc. 79, 39 [1953]. 